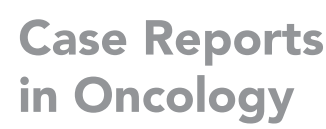

Case Reports

in Oncology

\title{
Sinonasal FUS-ERG-Rearranged Ewing's Sarcoma Mimicking Glomangiopericytoma
}

\author{
Maggie Zhou $\quad$ Yen Chen Kevin Ko ${ }^{\text {b }}$ Gregory W. Charville ${ }^{b}$ \\ Kristen N. Ganjoo ${ }^{c}$ \\ aDepartment of Medicine, Stanford University School of Medicine, Stanford, CA, USA; \\ bDepartment of Pathology, Stanford University School of Medicine, Stanford, CA, USA; \\ 'Department of Medicine (Oncology), Stanford University School of Medicine, Stanford, CA, \\ USA
}

\author{
Keywords \\ Ewing's sarcoma $\cdot$ Sinonasal $\cdot$ Genetic $\cdot$ Translocation
}

\begin{abstract}
Ewing's sarcoma is a rare and aggressive tumor that typically arises in the long bones of the extremities. It belongs in the family of small round blue cell tumors and is characterized immunohistochemically by diffuse CD99 expression and molecularly by one of several oncogenic translocations, most commonly $\mathrm{t}(11 ; 22)(\mathrm{q} 24 ; \mathrm{q} 12)$ between the EWSR1 gene and the FLI1 gene. Here we present a rare case of Ewing's sarcoma in the sinonasal tract with FUS-ERG gene arrangement that was regarded for almost a decade as a sinonasal-type hemangiopericytoma (glomangiopericytoma). This case illustrates the surprisingly prolonged natural history of Ewing's sarcoma that did not receive therapy for many years and the importance of considering alternative genetic translocations. Our experience suggests that the presence of diffuse CD99 membranous staining pattern in a small blue round cell tumor with morphology typical for Ewing's sarcoma but FISH negative for EWSR1 rearrangement should prompt consideration of FUS-ERG fusion.

(C) 2020 The Author(s).

Published by S. Karger AG, Basel
\end{abstract}




\section{Case Reports in Oncology}

\begin{tabular}{l|l}
\hline Case Rep Oncol 2020;13:1393-1396 \\
\hline DOI: 10.1159/000511415 & $\begin{array}{l}\text { @ 2 2020 The Author(s). Published by S. Karger AG, Basel } \\
\text { www.karger.com/cro }\end{array}$ \\
\hline
\end{tabular}

Zhou et al.: Sinonasal FUS-ERG-Rearranged Ewing's Sarcoma

\section{Introduction}

Ewing's sarcoma is a rare and aggressive tumor that typically presents in the skeletal form but may also present less frequently in the extraskeletal form. Ewing's sarcoma in the extraskeletal form in the head and neck is rare, and even more rare in the sinonasal tract [1]. Only case reports of sinonasal Ewing's sarcoma have been reported in the literature [2]. Due to the rarity of the disease, Ewing's sarcoma of the sinonasal tract is often misdiagnosed. Diagnosis is based on history, immunostaining, ultrastructural examination, and evaluation for common genetic translocations. Here we present the case of a sinonasal Ewing's sarcoma that was initially misdiagnosed as glomangiopericytoma and was ultimately found to be Ewing's sarcoma with a rare FUS-ERG rearrangement.

\section{Case Report}

The patient is a 57-year-old man who presented with 2 months of left nasal congestion and epistaxis. Magnetic resonance imaging (MRI) showed a 2.7-cm left nasal cavity mass extending into the left ethmoid sinus and medial left maxillary sinus. Endoscopic resection was performed, and histologic examination led to a diagnosis of glomangiopericytoma (sinonasal-type hemangiopericytoma). The tumor recurred 3.5 years later with a $4-\mathrm{cm}$ mass abutting the left orbit. Over 2 years following this recurrence, the patient underwent 4 additional endoscopic resections, all yielding morphologic features of glomangiopericytoma.

Approximately 6 years after initial presentation and 3 months after the most recent preceding endoscopic resection, the patient began volumetric-modulated arc radiotherapy to a sum of 66 Gy delivered in 33 fractions. Twenty-seven months after completion of radiotherapy (8.5 years after presentation), another endoscopic surgery was performed, again revealing recurrent disease. Immunohistochemical characterization of the recurrent tumor was notable for diffuse and strong expression of CD99 (membranous pattern), PAX7, and ERG, in keeping with Ewing's sarcoma and suggesting an underlying $E R G$ translocation (Fig. 1) $[3,4]$. Beta-catenin exhibited exclusively membranous and cytoplasmic localization, providing
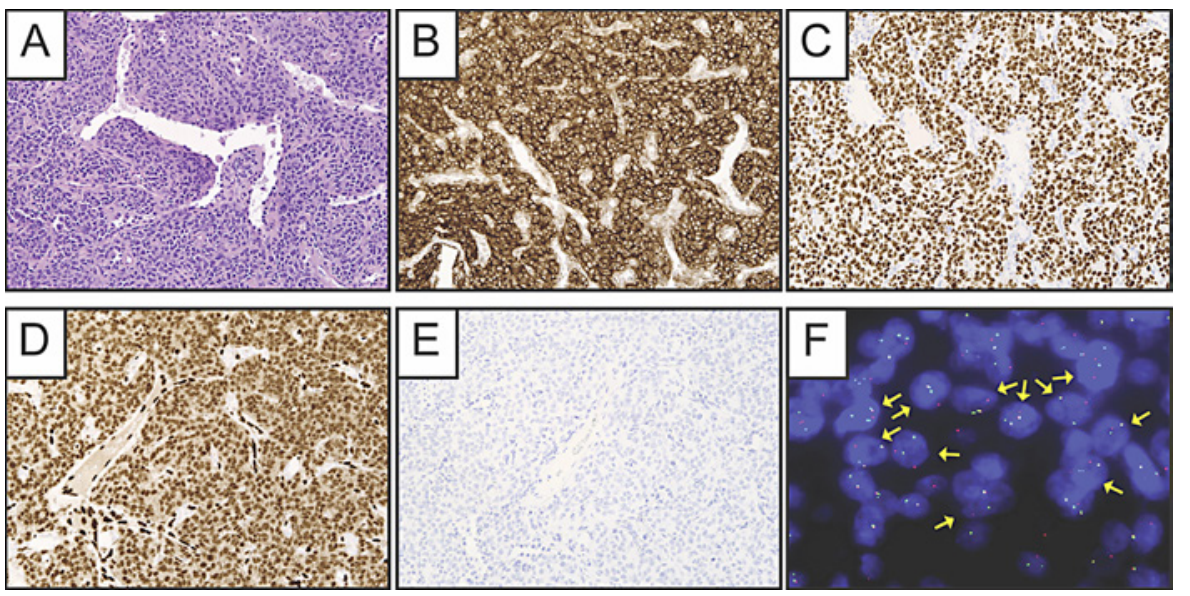

Fig. 1. H\&E-stained histologic sections of the sinonasal tumor revealed a proliferation of fairly monomorphic round cells with lightly eosinophilic cytoplasm and ill-defined cell borders in a background of prominent branching, thin-walled blood vessels (A). By immunohistochemistry, the neoplastic cells diffusely express CD99 (B), PAX7 (C), and ERG (D). There is no expression of cytokeratin 5/6 (E). Fluorescence in situ hybridization studies using break-apart probes showed FUS gene rearrangement (F). 
no indication of pathogenic beta-catenin signaling activation. Fluorescence in situ hybridization (FISH) studies using separation probes were notable for FUS gene rearrangement without EWSR1 rearrangement. Targeted RNA-sequencing subsequently confirmed an underlying pathogenic FUS-ERG translocation. FISH studies performed on the earliest endoscopic resection specimen were also indicative of FUS gene rearrangement.

The patient was treated 9 years after presentation with wedge resection of metastatic tumor to the right upper lung, exhibiting histologic features identical to those of the sinonasal tumor, and then underwent 6 cycles of temozolomide and irinotecan followed by maintenance temozolomide. Nine months after starting maintenance temozolomide (and 10 years after presentation), he developed recurrence of tumor in the left maxillary sinus, and temozolomide was stopped. He was started on pazopanib and remains on treatment at last clinical follow-up 11 years after presentation.

\section{Discussion}

We present a case of FUS-ERG-rearranged Ewing's sarcoma of the sinonasal area, which was regarded for almost a decade as a sinonasal-type hemangiopericytoma (glomangiopericytoma). This case illustrates the surprisingly prolonged natural history of an Ewing's sarcoma that did not receive "definitive" therapy for some time. Clinically and histologically, the tumor seems to fit with what has been previously described as an "adamantinoma-like Ewing family tumor" of the head and neck. The histopathologic diagnosis of this entity is often problematic due to a large number of potential mimics; therefore, in the microscopic evaluation of any poorly differentiated head and neck tumor, cellular monotony and CD99 immunoreactivity should prompt consideration of Ewing's sarcoma [5].

Ewing's sarcoma is a rare and aggressive neuroectodermal tumor that typically arises in the long bones of the extremities. Sinonasal Ewing family of tumors appear to be less aggressive than more typical extremity bone and soft tissue tumors; however, clinical outcome data are limited. Ewing's sarcoma is characterized immunohistochemically by diffuse CD99 expression and molecularly by one of several oncogenic translocations, most commonly $t(11 ; 22)$ (q24;q12) between the EWSR1 gene and the FLI1 gene. The second most common molecular abnormality is the $\mathrm{t}(21 ; 22)(\mathrm{q} 22 ; \mathrm{q} 12)$, which results in an EWSR1-ERG fusion. A small subset show EWSR1 fusions with other members of the ETS family of transcription factors, including ETV1 (7p22), E1A-F (17q21), and FEV (2q35-36) [6]. Other more rare fusions have been described in the literature and include EWSR1 being fused to non-ETS partners or FUS substituting for EWSR1. The limited data prevent drawing definitive conclusions as to whether these genetic subtypes should be treated as separate pathologic entities. EWSR1 and FUS are members of the FET family of RNA binding proteins and often interchange in translocationassociated sarcomas, but FUS rearrangements are rarely reported. In a retrospective case series of FUS-rearranged cases, patients were older and more evenly distributed between bone and soft tissue. Morphologically, FUS-ERG fusion-positive cases had similar monomorphic round cell cytomorphology to the classic phenotype and often showed diffuse membranous CD99 reactivity.

The current case offers a striking example of sinonasal Ewing's sarcoma with a prolonged natural history, even in the absence of "definitive" therapy. Our experience suggests that the presence of diffuse CD99 membranous staining pattern in a small blue round cell tumor with morphology typical for Ewing's sarcoma but FISH negative for EWSR1 rearrangement should prompt consideration of FUS-ERG fusion.

\section{Karger's}




\section{Case Reports in Oncology}

Case Rep Oncol 2020;13:1393-1396

\begin{tabular}{l|l}
\hline DOI: $10.1159 / 000511415$ & ○ 2020 The Author(s). Published by S. Karger AG, Basel
\end{tabular} www.karger.com/cro

Zhou et al.: Sinonasal FUS-ERG-Rearranged Ewing's Sarcoma

\section{Statement of Ethics}

Written informed consent was obtained from the patient for publication of this case report and any accompanying images.

\section{Conflict of Interest Statement}

The authors have no conflicts of interest to disclose.

\section{Funding Sources}

The authors did not receive any funding.

\section{Author Contributions}

M.Z. and Y.C.K.K. drafted the manuscript; M.Z., G.W.C., and K.N.G. revised the manuscript; all authors were substantially involved in the conception and design of the case report, as well as in the acquisition, analysis, and interpretation of the data; all authors approved the final version.

\section{References}

1 Negru ME, Sponghini AP, Rondonotti D, Platini F, Giavarra M, Forti L, et al. Primary Ewing's sarcoma of the sinonasal tract, eroding the ethmoid and sphenoid sinus with intracranial extension: A rare case report. Mol Clin Oncol. 2015 Jul;3(4):807-10.

2 Liang J. Sinonasal Ewing Sarcoma: A Case Report and Literature Review. Perm J [Internet]. 2018 Mar 7 [cited 2020 Aug 1].

3 Wang WL, Patel NR, Caragea M, Hogendoorn PC, López-Terrada D, Hornick JL, et al. Expression of ERG, an Ets family transcription factor, identifies ERG-rearranged Ewing sarcoma. Mod Pathol. 2012 Oct;25(10):1378-83.

4 Charville GW, Wang WL, Ingram DR, Roy A, Thomas D, Patel RM, et al. EWSR1 fusion proteins mediate PAX7 expression in Ewing sarcoma. Mod Pathol. 2017 Sep;30(9):1312-20.

5 Alexiev BA, Tumer Y, Bishop JA. Sinonasal adamantinoma-like Ewing sarcoma: A case report. Pathol Res Pract. 2017 Apr;213(4):422-6.

6 Chen S, Deniz K, Sung Y-S, Zhang L, Dry S, Antonescu CR. Ewing sarcoma with ERG gene rearrangements: A molecular study focusing on the prevalence of FUS-ERG and common pitfalls in detecting EWSR1-ERG fusions by FISH: Ewing Sarcoma With ERG Gene Rearrangements. Genes Chromosomes Cancer. 2016 Apr;55(4): 340-9.

\section{Karger'k}

